TRANSACTIONS OF THE

AMERICAN MATHEMATICAL SOCIETY

Volume 354, Number 4, Pages 1435-1452

S 0002-9947(01)02957-9

Article electronically published on December 4, 2001

\title{
WEAK AMENABILITY OF TRIANGULAR BANACH ALGEBRAS
}

\author{
B. E. FORREST AND L. W. MARCOUX
}

\begin{abstract}
Let $\mathcal{A}$ and $\mathcal{B}$ be unital Banach algebras, and let $\mathcal{M}$ be a Banach $\mathcal{A}, \mathcal{B}$-module. Then $\mathcal{T}=\left[\begin{array}{cc}\mathcal{A} & \mathcal{M} \\ 0 & \mathcal{B}\end{array}\right]$ becomes a triangular Banach algebra when equipped with the Banach space norm $\left\|\left[\begin{array}{cc}a & m \\ 0 & b\end{array}\right]\right\|=\|a\|_{\mathcal{A}}+\|m\|_{\mathcal{M}}+$ $\|b\|_{\mathcal{B}}$. A Banach algebra $\mathcal{T}$ is said to be $n$-weakly amenable if all derivations from $\mathcal{T}$ into its $n^{\text {th }}$ dual space $\mathcal{T}^{(n)}$ are inner. In this paper we investigate Arens regularity and $n$-weak amenability of a triangular Banach algebra $\mathcal{T}$ in relation to that of the algebras $\mathcal{A}, \mathcal{B}$ and their action on the module $\mathcal{M}$.
\end{abstract}

\section{INTRODUCTION}

In general, one can obtain a good deal of information about the structure of a Banach algebra $\mathcal{A}$ by studying the various Hochschild cohomology groups $H^{n}(\mathcal{A}, \mathcal{X})$ of $\mathcal{A}$ with coefficients in a Banach $\mathcal{A}$-bimodule $\mathcal{X}$. For example, the study of the first cohomology group $H^{1}(\mathcal{A}, \mathcal{X})$ is essentially the study of inner (vs. outer) derivations from $\mathcal{A}$ into $\mathcal{X}$, while if $\mathcal{X}$ is finite-dimensional, the study of $H^{2}(\mathcal{A}, \mathcal{X})$ is related to that of strong splittings of extensions of $\mathcal{A}$ by $\mathcal{X}[\underline{\mathbb{1}}$. For this reason, it is perhaps discouraging to know that explicit calculations of cohomology groups tends to be rather difficult, and results often focus on whether a given group $H^{n}(\mathcal{A}, \mathcal{X})$ is or is not trivial.

In 7], motivated by work of Gilfeather and Smith [8] in their study of joins of operator algebras, we began an investigation of derivations of triangular Banach algebras; these are algebras of the form

$$
\mathcal{T}=\left[\begin{array}{cc}
\mathcal{A} & \mathcal{M} \\
0 & \mathcal{B}
\end{array}\right]
$$

where $\mathcal{A}$ and $\mathcal{B}$ are themselves Banach algebras and $\mathcal{M}$ is a Banach $\mathcal{A}, \mathcal{B}$-module. In that paper a technique was developed which, in many cases, allows one to explicitly compute $H^{1}(\mathcal{T}, \mathcal{T})$ for specific choices of $\mathcal{A}, \mathcal{B}$, and $\mathcal{M}$.

In this paper, we show that the first cohomology group for these algebras with coefficients in their $n^{\text {th }}$ dual spaces $\mathcal{T}^{(n)}$ is also often tractable. In particular, we will develop criteria for deciding when $H^{1}\left(\mathcal{T}, \mathcal{T}^{(n)}\right)=0$. This notion, referred to as $n$-weak amenability and defined by Dales, Ghahramani and Grønbæk in [5], splits along two distinct lines in the setting of triangular Banach algebras. Indeed, when $n$ is odd, the (first) cohomology group of $\mathcal{T}$ depends only upon that of $\mathcal{A}$ and $\mathcal{B}$ [see Theorem [3.7 below]. When $n$ is even, the techniques developed in [7 can be

Received by the editors October 9, 1998 and, in revised form, July 20, 1999.

2000 Mathematics Subject Classification. Primary 46H25, 16E40.

Research supported in part by NSERC (Canada). 
extended to this setting, where it allows us to compute $H^{1}\left(\mathcal{T}, \mathcal{T}^{(2 n)}\right)$ for many choices of $\mathcal{A}, \mathcal{B}$ and $\mathcal{M}$ [see Theorem 3.12 .

The body of this paper is divided into three parts. In Section 2, we examine the natural actions of $\mathcal{T}$ on its dual spaces $\mathcal{T}^{(n)}, n \geq 1$, and characterize when $\mathcal{T}$ is Arens regular. In Section 3, we obtain our two main results concerning $H^{1}\left(\mathcal{T}, \mathcal{T}^{(n)}\right)$, the first dealing with the case where $n$ is odd, and the second dealing with the case where $n$ is even. Finally, in Section 4, we apply these results to three (classes) of examples, including tensor products of a given $C^{*}$-algebra $\mathcal{A}$ by $\mathcal{T}_{2}$, and finite dimensional nest algebras.

\section{Preliminaries}

2.1. Let $\mathcal{A}$ and $\mathcal{B}$ be Banach algebras, and suppose $\mathcal{X}$ is a Banach $\mathcal{A}, \mathcal{B}$-module; that is, $\mathcal{X}$ is a Banach space, a left $\mathcal{A}$-module and a right $\mathcal{B}$-module, and the actions of $\mathcal{A}$ and $\mathcal{B}$ are continuous in that

$$
\|a \circ x \circ b\| \leq\|a\|_{\mathcal{A}}\|x\|_{\mathcal{X}}\|b\|_{\mathcal{B}} .
$$

If $\mathcal{A}$ has a unit $e_{\mathcal{A}}$ and $\mathcal{B}$ has a unit $e_{\mathcal{B}}$, then $\mathcal{X}$ is said to be unital provided that $e_{\mathcal{A}} \circ x=x \circ e_{\mathcal{B}}=x$ for all $x \in \mathcal{X}$.

We can define a right action of $\mathcal{A}$ on the dual space $\mathcal{X}^{*}$ of $\mathcal{X}$ and a left action of $\mathcal{B}$ on $\mathcal{X}^{*}$ via

$$
\begin{aligned}
& (\phi \circ a)(x)=\phi(a \circ x), \\
& (b \circ \phi)(x)=\phi(x \circ b),
\end{aligned}
$$

for all $a \in \mathcal{A}, b \in \mathcal{B}, x \in \mathcal{X}$, and $\phi \in \mathcal{X}^{*}$.

Similarly, the second dual $\mathcal{X}^{(2)}$ of $\mathcal{X}$ becomes a Banach $\mathcal{A}, \mathcal{B}$-module under the actions

$$
\begin{aligned}
& (a \circ \Phi)(\phi)=\Phi(\phi \circ a), \\
& (\Phi \circ b)(\phi)=\Phi(b \circ \phi),
\end{aligned}
$$

for all $a \in \mathcal{A}, b \in \mathcal{B}, \phi \in \mathcal{X}^{*}$, and $\Phi \in \mathcal{X}^{(2)}$.

Of course, we may continue this process to higher order dual spaces of $\mathcal{X}$, with the conclusion being that $\mathcal{X}^{(2 m)}$ is a Banach $\mathcal{A}, \mathcal{B}$-module and $\mathcal{X}^{(2 m-1)}$ is a Banach $\mathcal{B}, \mathcal{A}$-module for all $m \geq 1$. When $\mathcal{X}$ is unital, so is each $\mathcal{X}^{(m)}, m \geq 1$. When $\mathcal{A}=\mathcal{B}$, we simply have that each $\mathcal{X}^{(m)}$ is a Banach $\mathcal{A}$-bimodule. A particular but important example of this is when $\mathcal{X}=\mathcal{A}=\mathcal{B}$, and the module actions are given by $a \circ x=a x$ and $x \circ a=x a$ for all $a, x \in \mathcal{A}$. The above analysis then shows that $\mathcal{A}^{(m)}$ is a Banach $\mathcal{A}$-bimodule for all $m \geq 1$.

Suppose $\mathcal{X}$ is a Banach $\mathcal{A}$-bimodule. A derivation $\delta: \mathcal{A} \rightarrow \mathcal{X}$ is a linear map which satisfies $\delta(a b)=\delta(a) \circ b+a \circ \delta(b)$ for all $a, b \in \mathcal{A}$. In this paper, we shall only consider continuous derivations. The derivation $\delta$ is said to be inner if there exists $x \in \mathcal{X}$ such that $\delta(a)=\delta_{x}(a):=a \circ x-x \circ a$ for all $a \in \mathcal{A}$. Denoting the linear space of bounded derivations from $\mathcal{A}$ into $\mathcal{X}$ by $Z^{1}(\mathcal{A}, \mathcal{X})$ and the linear subspace of (necessarily bounded) inner derivations by $N^{1}(\mathcal{A}, \mathcal{X})$, we may consider the quotient space $H^{1}(\mathcal{A}, \mathcal{X})=Z^{1}(\mathcal{A}, \mathcal{X}) / N^{1}(\mathcal{A}, \mathcal{X})$, called the first Hochschild cohomology group of $\mathcal{A}$ with coefficients in $\mathcal{X}$.

The Banach algebra $\mathcal{A}$ is said to be amenable if $H^{1}\left(\mathcal{A}, \mathcal{X}^{*}\right)=0$ for all Banach $\mathcal{A}$-bimodules $\mathcal{X}$. This notion was first defined by Johnson in [10], who showed that the group algebra $L^{1}(G)$ is amenable precisely when $G$ is an amenable group. In particular, the equivalence of amenability and nuclearity for $C^{*}$-algebras [9] 
indicates that amenability can be viewed as a generalized "finiteness" condition on a Banach algebra.

Bade, Curtis and Dales [1 later defined the notion of weak amenability for a commutative Banach algebra $\mathcal{A}$. They called $\mathcal{A}$ weakly amenable if every bounded derivation from $\mathcal{A}$ into a symmetric Banach $\mathcal{A}$-module is inner and hence zero. They also showed that this is equivalent to $H^{1}\left(\mathcal{A}, \mathcal{A}^{*}\right)=0$. This latter condition, that $H^{1}\left(\mathcal{A}, \mathcal{A}^{*}\right)=0$, has been adopted as the definition of weak amenability for an arbitrary Banach algebra. That this is strictly weaker than amenability is clear, since all $C^{*}$-algebras are weakly amenable 9 .

More recently, Dales, Ghahramani and Grønbæk [5] have defined the notions of $n$-weak amenability and permanent weak amenability for a Banach algebra $\mathcal{A}$ as follows: let $n$ be a positive integer. Then $\mathcal{A}$ is said to be $n$-weakly amenable if $H^{1}\left(\mathcal{A}, \mathcal{A}^{(n)}\right)=0$, and $\mathcal{A}$ is permanently weakly amenable if it is $n$-weakly amenable for all $n \geq 1$. Amongst other things, they were able to show that every $C^{*}$-algebra is permanently weakly amenable ([5], Theorem 2.1).

In this note we shall consider the $n$-weak amenability of a class of Banach algebras we first studied in [7, and which we call triangular Banach algebras. They are defined in the following way: let $\mathcal{A}$ and $\mathcal{B}$ be Banach algebras and suppose that $\mathcal{M}$ is an essential Banach $\mathcal{A}, \mathcal{B}$-module; that is, $\mathcal{A} \circ \mathcal{M}=\mathcal{M} \circ \mathcal{B}=\mathcal{M}$. (This is guaranteed when $\mathcal{M}$ is unital.) We define the corresponding triangular Banach algebra

$$
\mathcal{T}=\left[\begin{array}{cc}
\mathcal{A} & \mathcal{M} \\
& \mathcal{B}
\end{array}\right]
$$

with the sum and product being given by the usual $2 \times 2$ matrix operations and obvious internal module actions. The norm on $\mathcal{T}$ is

$$
\left\|\left[\begin{array}{cc}
a & m \\
& b
\end{array}\right]\right\|:=\|a\|_{\mathcal{A}}+\|m\|_{\mathcal{M}}+\|b\|_{\mathcal{B}}
$$

An important class of examples arises when $\mathcal{H}$ is a complex Hilbert space, $\mathcal{A}$ and $\mathcal{B}$ are operator subalgebras of $\mathcal{B}(\mathcal{H})$, and $\mathcal{M}$ is an essential $\mathcal{A}, \mathcal{B}$-submodule of $\mathcal{B}(\mathcal{H}) . \mathcal{T}$ is still not quite an operator subalgebra of $\mathcal{B}(\mathcal{H} \oplus \mathcal{H})$, however, since the given norm and the operator norm do not coincide.

2.2. Arens regularity for triangular Banach algebras. Given a triangular Banach algebra $\mathcal{T}$ as above, we can make $\mathcal{A}^{(2)}$ and $\mathcal{B}^{(2)}$ into Banach algebras by giving them the first Arens product as follows:

Let $\left\{a_{i}\right\},\left\{a_{j}\right\}$ be nets in $\mathcal{A}$ with $\Gamma_{1}=w^{*}-\lim _{i} a_{i}$ and $\Gamma_{2}=w^{*}-\lim _{j} a_{j}$. Then we define

$$
\Gamma_{1} \square \Gamma_{2}=w^{*}-\lim _{i} \lim _{j} a_{i} a_{j}
$$

Similarly, we can define

$$
\Psi_{1} \square \Psi_{2}=w^{*}-\lim _{i} \lim _{j} b_{i} b_{j}
$$

whenever $\Psi_{1}=w^{*}-\lim _{i} b_{i}$ and $\Psi_{2}=w^{*}-\lim _{j} b_{j}$. Moreover, we can extend the actions of $\mathcal{A}$ on $\mathcal{M}$ and of $\mathcal{B}$ on $\mathcal{M}$ to actions of $\mathcal{A}^{(2)}$ and $\mathcal{B}^{(2)}$ on $\mathcal{M}^{(2)}$ via

$$
\Gamma \square \Pi=w^{*}-\lim _{i} \lim _{j} a_{i} m_{j}
$$


and

$$
\Pi \square \Psi=w^{*}-\lim _{j} \lim _{k} m_{j} b_{k},
$$

where $\Gamma=w^{*}-\lim _{i} a_{i}, \quad \Pi=w^{*}-\lim _{j} m_{j}$, and $\Psi=w^{*}-\lim _{k} b_{k}$. This allows us to show that the first Arens multiplication defined on $\mathcal{T}^{(2)}$ behaves in a natural way.

Let $\left[\begin{array}{cc}\Gamma_{1} & \Pi_{1} \\ & \Psi_{1}\end{array}\right],\left[\begin{array}{cc}\Gamma_{2} & \Pi_{2} \\ & \Psi_{2}\end{array}\right] \in \mathcal{T}^{(2)}$. Assume also that

$$
\left[\begin{array}{ll}
\Gamma_{1} & \Pi_{1} \\
& \Psi_{1}
\end{array}\right]=w^{*}-\lim _{i}\left[\begin{array}{ll}
a_{i} & m_{i} \\
& b_{i}
\end{array}\right]
$$

and

$$
\left[\begin{array}{ll}
\Gamma_{2} & \Pi_{2} \\
& \Psi_{2}
\end{array}\right]=w^{*}-\lim _{j}\left[\begin{array}{ll}
a_{j} & m_{j} \\
& b_{j}
\end{array}\right]
$$

Then it is easy to see that $\Gamma_{1}=w^{*}-\lim _{i} a_{i}, \Gamma_{2}=w^{*}-\lim _{j} a_{j}, \Psi_{1}=w^{*}-\lim _{i} b_{i}, \Psi_{2}=$ $w^{*}-\lim _{j} b_{j}, \Pi_{1}=w^{*}-\lim _{i} m_{i}$ and $\Pi_{2}=w^{*}-\lim _{j} m_{j}$. Moreover,

$$
\begin{aligned}
{\left[\begin{array}{ll}
\Gamma_{1} & \Pi_{1} \\
& \Psi_{1}
\end{array}\right] \square\left[\begin{array}{ll}
\Gamma_{2} & \Pi_{2} \\
& \Psi_{2}
\end{array}\right]=w^{*}-\lim _{i} \lim _{j}\left[\begin{array}{ll}
a_{i} & m_{i} \\
& b_{i}
\end{array}\right]\left[\begin{array}{ll}
a_{j} & m_{j} \\
& b_{j}
\end{array}\right] } \\
=w^{*}-\lim _{i} \lim _{j}\left[\begin{array}{ll}
a_{i} a_{j} & a_{i} m_{j}+m_{i} b_{j} \\
& b_{i} b_{j}
\end{array}\right] \\
=\left[\begin{array}{ll}
w^{*}-\lim _{i} \lim _{j} a_{i} a_{j} & w^{*}-\lim _{i} \lim _{j}\left(a_{i} m_{j}+m_{i} b_{j}\right) \\
& w^{*}-\lim _{i} \lim _{j} b_{i} b_{j}
\end{array}\right] . \\
=\left[\begin{array}{ll}
\Gamma_{1} \square \Gamma_{2} & \Gamma_{1} \square \Pi_{2}+\Pi_{1} \square \Psi_{2} \\
\Psi_{1} \square \Psi_{2}
\end{array}\right] .
\end{aligned}
$$

Thus the first Arens product of $\mathcal{T}$ behaves just like matrix multiplication, with coordinate-level operations behaving like the first Arens product of the building blocks.

There is a second way of defining a product on the second dual of a Banach algebra, namely by interchanging the order of the limits taken in the first Arens product. The result of doing this is called the second Arens product.

We now set

$$
\Gamma_{1} \diamond \Gamma_{2}=w^{*}-\lim _{j} \lim _{i} a_{i} a_{j}
$$

and similarly,

$$
\begin{aligned}
& \Psi_{1} \diamond \Psi_{2}=w^{*}-\lim _{j} \lim _{i} b_{i} b_{j}, \\
& \Gamma_{1} \diamond \Pi=w^{*}-\lim _{j} \lim _{i} a_{i} m_{j}, \\
& \Pi \diamond \Psi_{1}=w^{*}-\lim _{i} \lim _{j} m_{j} b_{i} .
\end{aligned}
$$


The previous analysis of the first Arens product can now be extended to the second Arens product to see that

$$
\begin{aligned}
{\left[\begin{array}{cc}
\Gamma_{1} & \Pi_{1} \\
& \Psi_{1}
\end{array}\right] \diamond\left[\begin{array}{cc}
\Gamma_{2} & \Pi_{2} \\
& \Psi_{2}
\end{array}\right]=w^{*}-\lim _{j} \lim _{i}\left[\begin{array}{cc}
a_{i} & m_{i} \\
& b_{i}
\end{array}\right]\left[\begin{array}{cc}
a_{j} & m_{j} \\
& b_{j}
\end{array}\right] } \\
=w^{*}-\lim _{j} \lim _{i}\left[\begin{array}{cc}
a_{i} a_{j} & a_{i} m_{j}+m_{i} b_{j} \\
b_{i} b_{j}
\end{array}\right] \\
=\left[\begin{array}{cc}
w^{*}-\lim _{j} \lim _{i} a_{i} a_{j} & w^{*}-\lim _{j} \lim _{i}\left(a_{i} m_{j}+m_{i} b_{j}\right) \\
w^{*}-\lim _{j} \lim _{i} b_{i} b_{j}
\end{array}\right] \\
=\left[\begin{array}{cc}
\Gamma_{1} \diamond \Gamma_{2} & \Gamma_{1} \diamond \Pi_{2}+\Pi_{1} \diamond \Psi_{2} \\
\Psi_{1} \diamond \Psi_{2}
\end{array}\right] .
\end{aligned}
$$

In general, a Banach algebra $\mathcal{D}$ is said to be Arens regular provided that the first and second Arens products on $\mathcal{D}^{(2)}$ agree. Given $D, F \in \mathcal{D}^{(2)}$, it is known that $D \square F=D \diamond F$ whenever either $D$ or $F$ lies in the image of $\mathcal{D}$ under the canonical embedding of $\mathcal{D}$ into $\mathcal{D}^{(2)}$ ([13], Theorem 1.4.2) A fortiori, when this is the case, the Arens product also coincides with the natural dual module action of $\mathcal{D}$ on $\mathcal{D}^{(2)}$ defined at the beginning of Section 2.1. To see this, suppose for instance that $D, F \in \mathcal{D}^{(2)}$ with $D$ being the image of some $d \in \mathcal{D}$ under the canonical embedding. Suppose $F=w^{*}-\lim _{j} f_{j}$ and consider $\rho \in \mathcal{D}^{*}$. Then $(D \square F)=w^{*}-\lim _{j} d f_{j}$ and so

$$
(D \square F)(\rho)=\lim _{j} \rho\left(d f_{j}\right) .
$$

On the other hand, we have

$$
\begin{aligned}
(D \circ F)(\rho) & =F(\rho \circ d) \\
& =\lim _{j}(\rho \circ d)\left(f_{j}\right) \\
& =\lim _{j} \rho\left(d f_{j}\right) \\
& =(D \square F)(\rho) .
\end{aligned}
$$

The case where $F$ arises from the image of some $f \in \mathcal{D}$ under the canonical embedding and $D \in \mathcal{D}^{(2)}$ is arbitrary is handled in a similar fashion.

Returning to the setting of triangular Banach algebras, the same observations carry over to the case of the two $\mathcal{A}^{(2)}, \mathcal{B}^{(2)}$-module actions $\diamond$ and $\square$ which we have defined on $\mathcal{M}^{(2)}$. More precisely, $\Gamma \diamond \Pi=\Gamma \square \Pi$ if either $\Gamma$ is the image of some $a \in \mathcal{A}$ under the canonical embedding of $\mathcal{A}$ into $\mathcal{A}^{(2)}$, or if $\Pi$ is the image of some $m \in \mathcal{M}$ under the canonical embedding of $\mathcal{M}$ into its second dual. (A similar statement holds for the right action of $\mathcal{B}^{(2)}$ upon $\mathcal{M}^{(2)}$.) Again, when at least one term is the image of an element in $\mathcal{A}$ (or $\mathcal{M}$, or $\mathcal{B}$ ), these two actions $\square$ and $\diamond$ agree with the dual module action o defined above. We shall return to this later.

Let us say that the Banach algebras $\mathcal{A}$ and $\mathcal{B}$ act regularly on $\mathcal{M}$ if for every $\Gamma \in \mathcal{A}^{(2)}, \Pi \in \mathcal{M}^{(2)}$ and $\Psi \in \mathcal{B}^{(2)}$ we have

$$
\Gamma \square \Pi=\Gamma \diamond \Pi \quad \text { and } \quad \Pi \square \Psi=\Pi \diamond \Psi .
$$

The following proposition is now immediate:

2.3. Proposition. A triangular Banach algebra $\mathcal{T}=\left[\begin{array}{cc}\mathcal{A} & \mathcal{M} \\ & \mathcal{B}\end{array}\right]$ is Arens regular if and only if both $\mathcal{A}$ and $\mathcal{B}$ are Arens regular and $\mathcal{A}$ and $\mathcal{B}$ act regularly on $\mathcal{M}$. 
2.4. Now that we have shown that $\mathcal{T}^{(2)}$ can be made into a Banach algebra using either Arens product, we may apply the same argument to show that we can make $\mathcal{T}^{(4)}, \mathcal{T}^{(6)}$, and more generally $\mathcal{T}^{(2 n)}$ for $n \geq 1$ into a Banach algebra by repeatedly using the first (or second) Arens product. Two interesting implications of this analysis are the following:

(i) It provides us with an action of $\mathcal{T}$ upon $\mathcal{T}^{(2 n)}$ for each $n \geq 1$ via restriction of the Arens product to the canonical embedding of $\mathcal{T}$ into $\mathcal{T}^{(2 n)}$. As we have seen, this action agrees with the dual module action defined in Section 2.1.

(ii) The action of $\mathcal{T}$ upon $\mathcal{T}^{(2 n)}$ referred to in the previous paragraph in turn defines "actions" of $\mathcal{A}^{(2 n)}$ and $\mathcal{B}^{(2 n)}$ on $\mathcal{M}$ taking values in $\mathcal{M}^{(2 n)}$ via the formulae

$$
A \circ m=A \diamond \tilde{m} \quad \text { and } \quad m \circ B=\tilde{m} \diamond B
$$

where $A \in \mathcal{A}^{(2 n)}, B \in \mathcal{B}^{(2 n)}$, and $\tilde{m}$ denotes the (canonical) embedding of an element $m \in \mathcal{M}$ into $\mathcal{M}^{(2 n)}$. We shall use this in our study of derivations of $\mathcal{T}$ into $\mathcal{T}^{(2 n)}, n \geq 1$.

2.5. Iterated duals - the actions of $\mathcal{T}$ upon $\mathcal{T}^{(m)}$. Since, as a Banach space, $\mathcal{T}$ is isomorphic to the $\ell^{1}$-direct sum of $\mathcal{A}, \mathcal{M}$ and $\mathcal{B}$, it is clear that $\mathcal{T}^{(2 m-1)} \simeq$ $\mathcal{A}^{(2 m-1)} \oplus_{1} \mathcal{M}^{(2 m-1)} \oplus_{1} \mathcal{B}^{(2 m-1)}$, while $\mathcal{T}^{(2 m)} \simeq \mathcal{A}^{(2 m)} \oplus_{\infty} \mathcal{M}^{(2 m)} \oplus_{\infty} \mathcal{B}^{(2 m)}$ for each $m \geq 1$. Let us now consider the action of $\mathcal{T}$ upon $\mathcal{T}^{(m)}, m \geq 1$. To reduce notational clutter, we shall denote the module actions of $\mathcal{A}$ and $\mathcal{B}$ upon $\mathcal{M}$ simply by juxtaposition.

The CASE $m=1$. Suppose $t=\left[\begin{array}{cc}a & m \\ & b\end{array}\right] \in \mathcal{T}$ and $\tau=\left[\begin{array}{cc}\alpha & \mu \\ & \beta\end{array}\right] \in \mathcal{T}^{*}$. (Although we write $\tau$ as an upper triangular matrix, this is simply for convenience. The action of $\mathcal{T}^{*}$ upon $\mathcal{T}$ is given by $\tau(t)=\alpha(a)+\mu(m)+\beta(b)$.)

Let $w=\left[\begin{array}{ll}x & y \\ & z\end{array}\right] \in \mathcal{T}$. From above, the action of $w$ on $\tau$ is given by

$$
\begin{aligned}
(w \circ \tau)(t) & =\tau(t w) \\
& =\tau\left[\begin{array}{cc}
a x & a y+m z \\
b z
\end{array}\right] \\
& =\alpha(a x)+\mu(a y+m z)+\beta(b z) \\
& =(x \circ \alpha)(a)+(y \circ \mu)(a)+(z \circ \mu)(m)+(z \circ \beta)(b) \\
& =\left[\begin{array}{cc}
x \circ \alpha+y \circ \mu & z \circ \mu \\
& z \circ \beta
\end{array}\right]\left(\begin{array}{cc}
a & m \\
& b
\end{array}\right) .
\end{aligned}
$$

Thus $(w \circ \tau)=\left[\begin{array}{cc}x \circ \alpha+y \circ \mu & z \circ \mu \\ & z \circ \beta\end{array}\right]$.

Similarly, one can check that $(\tau \circ w)=\left[\begin{array}{cc}\alpha \circ x & \mu \circ x \\ & \mu \circ y+\beta \circ z\end{array}\right]$.

The CASE $m=2$. We can identify $\mathcal{T}^{(2)}$ with $\left[\begin{array}{c}\mathcal{A}^{(2)} \\ \mathcal{M}^{(2)} \\ \mathcal{B}^{(2)}\end{array}\right]$. As we have seen, the action of $\mathcal{T}$ upon $\mathcal{T}^{(2)}$ coincides with the restriction of the (first or second) Arens product on $\mathcal{T}^{(2)}$ to the image of $\mathcal{T}$ in $\mathcal{T}^{(2)}$ under the canonical embedding, and hence

$$
\left[\begin{array}{ll}
x & y \\
& z
\end{array}\right] \circ\left[\begin{array}{cc}
\Gamma & \Pi \\
& \Phi
\end{array}\right]=\left[\begin{array}{cc}
x \circ \Gamma & x \circ \Pi+y \circ \Phi \\
z \circ \Phi
\end{array}\right]
$$


and

$$
\left[\begin{array}{cc}
\Gamma & \Pi \\
& \Phi
\end{array}\right] \circ\left[\begin{array}{ll}
x & y \\
& z
\end{array}\right]=\left[\begin{array}{cc}
\Gamma \circ x & \Gamma \circ y+\Pi \circ z \\
\Phi \circ z
\end{array}\right] .
$$

Again, we observe that these equations coincide with the standard matrix "multiplications" of $w$ and $T$, with multiplication at the coordinate level corresponding to the appropriate module action.

THE CASE $m \geq 3$. It is not hard to see that the action of $\mathcal{T}$ on $\mathcal{T}^{(3)}$ agrees with the dual action of $\mathcal{T}^{(2)}$ on $\mathcal{T}^{(3)}$ when we restrict to the image of $\mathcal{T}$ in $\mathcal{T}^{(2)}$ under the canonical embedding. Indeed, note that if $\left[\begin{array}{cc}\theta & \eta \\ & \omega\end{array}\right] \in \mathcal{T}^{(3)},\left[\begin{array}{cc}\Gamma & \Pi \\ & \Psi\end{array}\right] \in \mathcal{T}^{(2)}$, and $\left[\begin{array}{cc}a & m \\ & b\end{array}\right] \in \mathcal{T}$, then

$$
\begin{aligned}
{\left[\begin{array}{cc}
\theta & \eta \\
& \omega
\end{array}\right] \circ\left[\begin{array}{cc}
a & m \\
& b
\end{array}\right]\left(\begin{array}{ll}
\Gamma & \Pi \\
& \Psi
\end{array}\right) } & =\left[\begin{array}{ll}
\theta & \eta \\
& \omega
\end{array}\right]\left(\left[\begin{array}{cc}
a & m \\
& b
\end{array}\right] \circ\left[\begin{array}{cc}
\Gamma & \Pi \\
& \Psi
\end{array}\right]\right) \\
& =\left[\begin{array}{ll}
\theta & \eta \\
& \omega
\end{array}\right]\left(\left[\begin{array}{cc}
a & m \\
& b
\end{array}\right] \diamond\left[\begin{array}{cc}
\Gamma & \Pi \\
& \Psi
\end{array}\right]\right)
\end{aligned}
$$

where $\circ$ denotes the appropriate dual action and $\diamond$ denotes the Arens product (recall that both Arens products agree since one of the terms is the image of an element of $\mathcal{T}$ under the canonical embedding). More generally, it follows that the computation of the action of $\mathcal{T}$ upon $\mathcal{T}^{*}$ carries over mutatis mutandis to the action of $\mathcal{T}$ upon $\mathcal{T}^{(2 m-1)}, m \geq 2$. Similarly, the action of $\mathcal{T}$ upon $\mathcal{T}^{(2 m)}, m \geq 2$, also satisfies the corresponding formulae for the action of $\mathcal{T}$ upon $\mathcal{T}^{(2)}$, and hence "looks like" standard matrix multiplication, as we saw in Section 2.4

\section{Derivations AND $n$-WeAK AMEnABiLity}

3.1. We now wish to consider the question of $n$-weak amenability of triangular Banach algebras $\mathcal{T}=\left[\begin{array}{cc}\mathcal{A} & \mathcal{M} \\ & \mathcal{B}\end{array}\right]$. From this point on, we shall assume that the corner algebras $\mathcal{A}$ and $\mathcal{B}$ are unital and that $\mathcal{M}$ is a unital Banach $\mathcal{A}, \mathcal{B}$-module. Although this is not crucial for weak amenability (i.e. when $n=1$ ), it guarantees that $\mathcal{M}^{(n)}$ is an essential module when $n \geq 2$. As we shall see, there are two distinct phenomena which occur, depending upon whether $n$ is even or odd. We begin by considering the case where $n$ is odd, and in fact, first considering the case where $n=1$.

Weak amenability. The proof of the following lemma involves nothing more than routine calculations which are left to the reader.

3.2. Lemma. Let $D: \mathcal{T} \rightarrow \mathcal{T}^{*}$ be a continuous derivation. Then there exist continuous derivations $\delta_{1}: \mathcal{A} \rightarrow \mathcal{A}^{*}, \delta_{4}: \mathcal{B} \rightarrow \mathcal{B}^{*}$, and an element $\gamma_{\delta} \in \mathcal{M}^{*}$ such that
(i) $D\left[\begin{array}{ll}x & 0 \\ & 0\end{array}\right]=\left[\begin{array}{cc}\delta_{1}(x) & \gamma_{\delta} \circ x \\ 0\end{array}\right] \quad$ for all $x \in \mathcal{A}$,$$
\begin{aligned}
& D\left[\begin{array}{ll}
0 & 0 \\
& z
\end{array}\right]=\left[\begin{array}{cc}
0 & -z \circ \gamma_{\delta} \\
\delta_{4}(z)
\end{array}\right] \quad \text { for all } z \in \mathcal{B} \text {, } \\
& D\left[\begin{array}{ll}
0 & y \\
& 0
\end{array}\right]=\left[\begin{array}{cc}
-y \circ \gamma_{\delta} & 0 \\
& \gamma_{\delta} \circ y
\end{array}\right] \quad \text { for all } y \in \mathcal{M} \text {. }
\end{aligned}
$$ 
3.3. Lemma. Suppose $\delta_{\mathcal{A}}: \mathcal{A} \rightarrow \mathcal{A}^{*}$ is a continuous derivation. Then

$$
\begin{aligned}
& D_{\delta_{\mathcal{A}}}: \quad \mathcal{T} \quad \rightarrow \quad \mathcal{T}^{*}, \\
& {\left[\begin{array}{cc}
a & m \\
& b
\end{array}\right] \mapsto\left[\begin{array}{cc}
\delta_{\mathcal{A}}(a) & 0 \\
& 0
\end{array}\right]}
\end{aligned}
$$

is also a continuous derivation. Furthermore, $\delta_{\mathcal{A}}$ is inner if and only if $D_{\delta_{\mathcal{A}}}$ is inner.

Similarly, suppose $\delta_{\mathcal{B}}: \mathcal{B} \rightarrow \mathcal{B}^{*}$ is a continuous derivation. Then

$$
D_{\delta_{\mathcal{B}}}: \begin{gathered}
\mathcal{T} \\
{\left[\begin{array}{cc}
a & m \\
& b
\end{array}\right]}
\end{gathered} \stackrel{\mapsto}{\mapsto}\left[\begin{array}{cc}
\mathcal{T}^{*}, & 0 \\
& \delta_{\mathcal{B}}(b)
\end{array}\right],
$$

is a continuous derivation, and it is inner precisely when $\delta_{\mathcal{B}}$ is inner.

Proof. That $D_{\delta_{\mathcal{A}}}$ and $D_{\delta_{\mathcal{B}}}$ are derivations are again elementary calculations which we leave to the reader.

Suppose $\delta_{\mathcal{A}}$ is inner. Then there exists $\alpha \in \mathcal{A}^{*}$ such that $\delta_{\mathcal{A}}(a)=\delta_{\alpha}(a):=$ $a \circ \alpha-\alpha \circ a$.

$$
\begin{aligned}
& \text { Consider } \tau=\left[\begin{array}{ll}
\alpha & 0 \\
& 0
\end{array}\right] \in \mathcal{T}^{*} \text {. Then } \\
& D_{\tau}\left[\begin{array}{cc}
a & m \\
& b
\end{array}\right]=\left[\begin{array}{cc}
a & m \\
& b
\end{array}\right] \circ\left[\begin{array}{ll}
\alpha & 0 \\
& 0
\end{array}\right]-\left[\begin{array}{ll}
\alpha & 0 \\
& 0
\end{array}\right] \circ\left[\begin{array}{cc}
a & m \\
& b
\end{array}\right] \\
& =\left[\begin{array}{cc}
a \circ \alpha-\alpha \circ a & 0 \\
& 0
\end{array}\right] \\
& =\left[\begin{array}{ll}
\delta_{\alpha}(a) & 0 \\
& 0
\end{array}\right] \\
& =D_{\delta_{\mathcal{A}}}\left[\begin{array}{cc}
a & m \\
& b
\end{array}\right] \text {, }
\end{aligned}
$$

and so $D_{\delta_{\mathcal{A}}}$ is inner.

Conversely, suppose $D_{\delta_{\mathcal{A}}}$ is inner. Then there exists $\tau=\left[\begin{array}{cc}\alpha & \mu \\ & \beta\end{array}\right] \in \mathcal{T}^{*}$ such that

$$
\begin{aligned}
D_{\delta_{\mathcal{A}}}\left[\begin{array}{cc}
a & m \\
& b
\end{array}\right] & =\left[\begin{array}{cc}
a & m \\
& b
\end{array}\right] \circ\left[\begin{array}{cc}
\alpha & \mu \\
\beta
\end{array}\right]-\left[\begin{array}{cc}
\alpha & \mu \\
\beta
\end{array}\right] \circ\left[\begin{array}{cc}
a & m \\
& b
\end{array}\right] \\
& =\left[\begin{array}{cc}
a \circ \alpha+m \circ \mu-\alpha \circ a \circ \mu-\mu \circ a \\
b \circ \beta-\mu \circ m-\beta \circ b
\end{array}\right] .
\end{aligned}
$$

But

$$
D_{\delta_{\mathcal{A}}}\left[\begin{array}{cc}
a & m \\
& b
\end{array}\right]=\left[\begin{array}{cc}
\delta_{\mathcal{A}}(a) & 0 \\
& 0
\end{array}\right]
$$

It follows that $\delta_{\mathcal{A}}(a)=a \circ \alpha-\alpha \circ a+m \circ \mu$ for all $a \in \mathcal{A}, m \in \mathcal{M}$. We may assume without loss of generality that $m=0$. Then for any $a \in \mathcal{A}$, we have

$$
\delta_{\mathcal{A}}(a)=a \circ \alpha-\alpha \circ a,
$$

and so $\delta_{\mathcal{A}}=\delta_{\alpha}$ is inner, as claimed. The proofs for $\delta_{\mathcal{B}}$ and $D_{\delta_{\mathcal{B}}}$ are similar. 
We mentioned in the above proof that the calculation that shows that derivations from the corner algebra $\mathcal{A}$ into its dual space lift to derivations from $\mathcal{T}$ into $\mathcal{T}^{*}$ is elementary. It is worth pointing out that the conclusion is false if we consider lifting derivations from $\mathcal{A}$ into $\mathcal{A}$ to derivations from $\mathcal{T}$ into $\mathcal{T}$. See [7], Example 3.6 for a counterexample.

3.4. Theorem. Let $\mathcal{A}$ and $\mathcal{B}$ be unital Banach algebras and $\mathcal{M}$ be a unital Banach $\mathcal{A}, \mathcal{B}$-module. Let $\mathcal{T}=\left[\begin{array}{cc}\mathcal{A} & \mathcal{M} \\ & \mathcal{B}\end{array}\right]$ be the corresponding triangular Banach algebra. Then

$$
H^{1}\left(\mathcal{T}, \mathcal{T}^{*}\right) \simeq H^{1}\left(\mathcal{A}, \mathcal{A}^{*}\right) \oplus H^{1}\left(\mathcal{B}, \mathcal{B}^{*}\right)
$$

Proof. Let $\delta: \mathcal{T} \rightarrow \mathcal{T}^{*}$ be a (continuous) derivation. From Lemma 3.2 above, we obtain derivations $\delta_{1}: \mathcal{A} \rightarrow \mathcal{A}^{*}$ and $\delta_{4}: \mathcal{B} \rightarrow \mathcal{B}^{*}$, as well as an element $\gamma_{\delta} \in \mathcal{M}^{*}$ such that

$$
\delta\left[\begin{array}{ll}
x & y \\
& z
\end{array}\right]=\left[\begin{array}{ll}
\delta_{1}(x)-y \circ \gamma_{\delta} & \gamma_{\delta} \circ x-z \circ \gamma_{\delta} \\
& \gamma_{\delta} \circ y+\delta_{4}(z)
\end{array}\right]
$$

Consider the linear map

$$
\begin{array}{ccc}
\kappa: \quad Z^{1}\left(\mathcal{T}, \mathcal{T}^{*}\right) & \rightarrow & H^{1}\left(\mathcal{A}, \mathcal{A}^{*}\right) \oplus H^{1}\left(\mathcal{B}, \mathcal{B}^{*}\right) \\
\delta & \mapsto & \left(\delta_{1}+N^{1}\left(\mathcal{A}, \mathcal{A}^{*}\right), \delta_{4}+N^{1}\left(\mathcal{B}, \mathcal{B}^{*}\right)\right)
\end{array}
$$

Clearly $\kappa$ is linear.

We claim that $\kappa$ is surjective. Indeed, given $\delta_{\mathcal{A}} \in Z^{1}\left(\mathcal{A}, \mathcal{A}^{*}\right)$ and $\delta_{\mathcal{B}} \in Z^{1}\left(\mathcal{B}, \mathcal{B}^{*}\right)$, Lemma 3.3 shows that the lifted maps $D_{\delta_{\mathcal{A}}}$ and $D_{\delta_{\mathcal{B}}}$ both lie in $Z^{1}\left(\mathcal{T}, \mathcal{T}^{*}\right)$, and thus $D=D_{\delta_{\mathcal{A}}}+D_{\delta_{\mathcal{B}}} \in Z^{1}\left(\mathcal{T}, \mathcal{T}^{*}\right)$. But then

$$
\begin{aligned}
\kappa(D) & =\kappa\left(D_{\delta_{\mathcal{A}}}\right)+\kappa\left(D_{\delta_{\mathcal{B}}}\right) \\
& =\left(\delta_{\mathcal{A}}+N^{1}\left(\mathcal{A}, \mathcal{A}^{*}\right), 0\right)+\left(0, \delta_{\mathcal{B}}+N^{1}\left(\mathcal{B}, \mathcal{B}^{*}\right)\right) \\
& =\left(\delta_{\mathcal{A}}+N^{1}\left(\mathcal{A}, \mathcal{A}^{*}\right), \delta_{\mathcal{B}}+N^{1}\left(\mathcal{B}, \mathcal{B}^{*}\right)\right) .
\end{aligned}
$$

Thus $\kappa$ is onto, as claimed.

Next, suppose $\delta \in \operatorname{ker} \kappa$. Then $\delta_{1} \in N^{1}\left(\mathcal{A}, \mathcal{A}^{*}\right)$ and $\delta_{4} \in N^{1}\left(\mathcal{B}, \mathcal{B}^{*}\right)$. By Lemma [3.3, $D_{\delta_{1}}, D_{\delta_{4}} \in N^{1}\left(\mathcal{T}, \mathcal{T}^{*}\right)$, and hence $D=D_{\delta_{1}}+D_{\delta_{4}} \in N^{1}\left(\mathcal{T}, \mathcal{T}^{*}\right)$. But $\delta-D=\delta\left[\begin{array}{cc}0 & \gamma_{\delta} \\ & 0\end{array}\right] \in N^{1}\left(\mathcal{T}, \mathcal{T}^{*}\right)$, and so $\delta$ is an inner derivation. That $\delta \in N^{1}\left(\mathcal{T}, \mathcal{T}^{*}\right)$ implies $\delta_{1}, \delta_{4}$ are also inner is again Lemma 3.3 Thus ker $\kappa=$ $N^{1}\left(\mathcal{T}, \mathcal{T}^{*}\right)$

Elementary linear algebra theory now implies that

$$
H^{1}\left(\mathcal{A}, \mathcal{A}^{*}\right) \oplus H^{1}\left(\mathcal{B}, \mathcal{B}^{*}\right)=\operatorname{ran} \kappa \simeq Z^{1}\left(\mathcal{T}, \mathcal{T}^{*}\right) / \operatorname{ker} \kappa=H^{1}\left(\mathcal{T}, \mathcal{T}^{*}\right) .
$$

3.5. Corollary. Let $\mathcal{A}$ and $\mathcal{B}$ be unital Banach algebras and $\mathcal{M}$ be a unital Banach $\mathcal{A}, \mathcal{B}$-module. Let $\mathcal{T}=\left[\begin{array}{cc}\mathcal{A} & \mathcal{M} \\ & \mathcal{B}\end{array}\right]$ be the corresponding triangular Banach algebra. Then $\mathcal{T}$ is weakly amenable if and only if both $\mathcal{A}$ and $\mathcal{B}$ are weakly amenable. 
3.6. Recall that the action of $\mathcal{T}$ on $\mathcal{T}^{(3)}$ is a restriction of the action of $\mathcal{T}^{(2)}$ on $\mathcal{T}^{(3)}$. From this we can again determine the behaviour of a derivation $D: \mathcal{T} \rightarrow \mathcal{T}^{(3)}$. As before,

$$
\left[\begin{array}{ll}
\theta & \eta \\
& \omega
\end{array}\right] \circ\left[\begin{array}{cc}
a & m \\
& b
\end{array}\right]=\left[\begin{array}{cc}
\theta \circ a & \eta \circ a \\
& \eta \circ m+\omega \circ b
\end{array}\right]
$$

and

$$
\left[\begin{array}{cc}
a & m \\
& b
\end{array}\right] \circ\left[\begin{array}{cc}
\theta & \eta \\
& \omega
\end{array}\right]=\left[\begin{array}{cc}
a \circ \theta+m \circ \eta & b \circ \eta \\
& b \circ \omega
\end{array}\right] .
$$

By repeating exactly the same calculations as for derivations $\delta$ from $\mathcal{T}$ into $\mathcal{T}^{*}$, we find that there exist derivations $\delta_{1}: \mathcal{A} \rightarrow \mathcal{A}^{(3)}$ and $\delta_{4}: \mathcal{B} \rightarrow \mathcal{B}^{(3)}$ as well as an element $\gamma_{\delta} \in \mathcal{M}^{(3)}$ (arising as the $(1,2)$-coordinate of the image of $\left.\delta\left(\left[\begin{array}{ll}e_{\mathcal{A}} & 0 \\ & 0\end{array}\right]\right)\right)$ ) such that

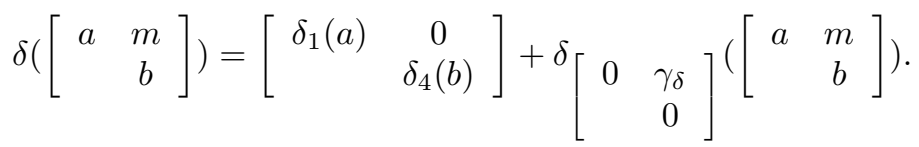

This shows that $H^{1}\left(\mathcal{T}, \mathcal{T}^{(3)}\right) \simeq H^{1}\left(\mathcal{A}, \mathcal{A}^{(3)}\right) \oplus H^{1}\left(\mathcal{B}, \mathcal{B}^{(3)}\right)$, and that, in particular, $\mathcal{T}$ is 3 -weakly amenable if and only if both $\mathcal{A}$ and $\mathcal{B}$ are. In fact, this argument extends to any dual of the form $\mathcal{T}^{(2 n-1)}$ with $n \geq 1$.

We therefore have:

3.7. Theorem. Let $\mathcal{A}$ and $\mathcal{B}$ be unital Banach algebras and $\mathcal{M}$ be a unital Banach $\mathcal{A}, \mathcal{B}$-module. Let $\mathcal{T}=\left[\begin{array}{cc}\mathcal{A} & \mathcal{M} \\ & \mathcal{B}\end{array}\right]$ be the corresponding triangular Banach algebra. Suppose $n$ is a positive integer. Then

$$
H^{1}\left(\mathcal{T}, \mathcal{T}^{(2 n-1)}\right) \simeq H^{1}\left(\mathcal{A}, \mathcal{A}^{(2 n-1)}\right) \oplus H^{1}\left(\mathcal{B}, \mathcal{B}^{(2 n-1)}\right) .
$$

It follows that $\mathcal{T}$ is $(2 n-1)$ - weakly amenable if and only if both $\mathcal{A}$ and $\mathcal{B}$ are.

3.8. (2n)-weak amenability. The study of derivations from $\mathcal{T}$ into $\mathcal{T}^{(2 n)}$ differs radically from that of derivations into $\mathcal{T}^{(2 n-1)}, n \geq 1$. While the $(2 n-1)$-weak amenability of $\mathcal{T}$ depends only upon the $(2 n-1)$-weak amenability of $\mathcal{A}$ and $\mathcal{B}$, the situation for $(2 n)$-weak amenability more closely resembles the situation explored in 7] for derivations from $\mathcal{T}$ into itself. Indeed, given that the action of $\mathcal{T}$ upon $\mathcal{T}^{(2 n)}$ mimics that of matrix multiplication, many of the calculations from that paper formally carry over intact to prove an analogue of [7], Thm. 2.8. For the sake of completeness, we shall include a detailed outline of the proof here, elaborating on the differences which occur as a result of having changed the codomain of the derivations.

3.9. Proposition. Let $n$ be a positive integer and suppose $\delta: \mathcal{T} \rightarrow \mathcal{T}^{(2 n)}$ is a continuous derivation. Then there exist $\gamma_{\delta} \in \mathcal{M}^{(2 n)}$, and continuous derivations $\delta_{1}: \mathcal{A} \rightarrow \mathcal{A}^{(2 n)}, \delta_{4}: \mathcal{B} \rightarrow \mathcal{B}^{(2 n)}$ and a continuous map $\rho: \mathcal{M} \rightarrow \mathcal{M}^{(2 n)}$ which satisfy:

(i) $\delta\left[\begin{array}{ll}a & 0 \\ & 0\end{array}\right]=\left[\begin{array}{cc}\delta_{1}(a) & a \circ \gamma_{\delta} \\ 0\end{array}\right] ;$
(ii) $\delta\left[\begin{array}{ll}0 & 0 \\ & b\end{array}\right]=\left[\begin{array}{cc}0 & -\gamma_{\delta} \circ b \\ \delta_{4}(b)\end{array}\right] ;$ 
(iii) $\delta\left[\begin{array}{cc}0 & m \\ & 0\end{array}\right]=\left[\begin{array}{cc}0 & \rho(m) \\ & 0\end{array}\right]$;

(iv) $\rho(a \circ m)=\delta_{1}(a) \circ m+a \circ \rho(m)$;

(v) $\rho(m \circ b)=\rho(m) \circ b+m \circ \delta_{4}(b)$.

(vi) Futhermore, if $\delta_{\mathcal{A}}: \mathcal{A} \rightarrow \mathcal{A}^{(2 n)}$ and $\delta_{\mathcal{B}}: \mathcal{B} \rightarrow \mathcal{B}^{(2 n)}$ are continuous derivations and $\rho_{\mathcal{M}}: \mathcal{M} \rightarrow \mathcal{M}^{(2 n)}$ is a continuous linear map that satisfies (iv) and (v), then

$$
\Delta\left[\begin{array}{cc}
a & m \\
& b
\end{array}\right]:=\left[\begin{array}{cc}
\delta_{\mathcal{A}}(a) & \rho_{\mathcal{M}}(m) \\
& \delta_{\mathcal{B}}(b)
\end{array}\right]
$$

defines a continuous derivation of $\mathcal{T}$ into $\mathcal{T}^{(2 n)}$.

Proof. The proofs of these statements rely on elementary matrix calculations and are left to the reader.

3.10. Given a Banach $\mathcal{A}, \mathcal{B}$-module $\mathcal{M}$ as we have, let us consider maps of the form

$$
\begin{array}{rlrl}
\rho_{x, z}: \mathcal{M} & \rightarrow & \mathcal{M}^{(2 n)}, \\
m & \mapsto x \circ m-m \circ z,
\end{array}
$$

where $x \in \mathcal{A}^{(2 n)}, z \in \mathcal{B}^{(2 n)}$. We shall denote the centralizer of $\mathcal{A}$ in $\mathcal{A}^{(2 n)}$ as $Z_{\mathcal{A}}\left(\mathcal{A}^{(2 n)}\right)=\left\{x \in \mathcal{A}^{(2 n)}: x \circ a=a \circ x\right.$ for all $\left.a \in \mathcal{A}\right\}$, and similarly, $Z_{\mathcal{B}}\left(\mathcal{B}^{(2 n)}\right)=$ $\left\{z \in \mathcal{B}^{(2 n)}: z \circ b=b \circ z\right.$ for all $\left.b \in \mathcal{B}\right\}$. In analogy with [7, we shall refer to the set

$$
Z R_{\mathcal{A}, \mathcal{B}}\left(\mathcal{M}, \mathcal{M}^{(2 n)}\right)=\left\{\rho_{x, z}: \mathcal{M} \rightarrow \mathcal{M}^{(2 n)} ; x \in Z_{\mathcal{A}}\left(\mathcal{A}^{(2 n)}\right), z \in Z_{\mathcal{B}}\left(\mathcal{B}^{(2 n)}\right\}\right.
$$

as central Rosenblum operators on $\mathcal{M}$ with coefficients in $\mathcal{M}^{(2 n)}$.

We also define

$$
\begin{gathered}
\operatorname{Hom}_{\mathcal{A}, \mathcal{B}}\left(\mathcal{M}, \mathcal{M}^{(2 n)}\right)=\left\{\phi: \mathcal{M} \rightarrow \mathcal{M}^{(2 n)} ; \phi(a \circ m)=a \circ \phi(m), \phi(m \circ b)\right. \\
=\phi(m) \circ b \text { for all } a \in \mathcal{A}, m \in \mathcal{M}, b \in \mathcal{B}\} .
\end{gathered}
$$

We then have

3.11. Lemma. (i) $Z R_{\mathcal{A}, \mathcal{B}}\left(\mathcal{M}, \mathcal{M}^{(2 n)}\right) \subseteq \operatorname{Hom}_{\mathcal{A}, \mathcal{B}}\left(\mathcal{M}, \mathcal{M}^{(2 n)}\right)$.

(ii) If $\phi \in \operatorname{Hom}_{\mathcal{A}, \mathcal{B}}\left(\mathcal{M}, \mathcal{M}^{(2 n)}\right)$, then the map

$$
\Delta_{\phi}\left[\begin{array}{cc}
a & m \\
& b
\end{array}\right]=\left[\begin{array}{cc}
0 & \phi(m) \\
& 0
\end{array}\right] \in Z^{1}\left(\mathcal{T}, \mathcal{T}^{(2 n)}\right) .
$$

Moreover, $\Delta_{\phi}$ is inner if and only if $\phi$ is a central Rosenblum operator on $\mathcal{M}$ with coefficients in $\mathcal{M}^{(2 n)}$.

Proof. (i) Suppose $\rho_{x, z} \in Z R_{\mathcal{A}, \mathcal{B}}\left(\mathcal{M}, \mathcal{M}^{(2 n)}\right)$, and that $a \in \mathcal{A}, b \in \mathcal{B}$. Then

$$
\begin{aligned}
\rho_{x, z}(a \circ m) & =x \circ(a \circ m)-(a \circ m) \circ z \\
& =(x \circ a) \circ m-a \circ(m \circ z) \\
& =(a \circ x) \circ m-a \circ(m \circ z) \\
& =a \circ(x \circ m-m \circ z) \\
& =a \circ \rho_{x, z}(m) .
\end{aligned}
$$

Similarly, $\rho_{x, z}(m \circ b)=\rho_{x, z}(m) \circ b$. 
(ii) The first statement is Proposition $3.9[(\mathrm{vi})]$ applied to the case where $\delta_{\mathcal{A}}=$ $\delta_{\mathcal{B}}=0$. Suppose $\phi=\rho_{x, z} \in \operatorname{Hom}_{\mathcal{A}, \mathcal{B}}\left(\mathcal{M}, \mathcal{M}^{(2 n)}\right)$. Then

$$
\begin{aligned}
\delta_{[}\left[\begin{array}{ll}
x & 0 \\
& z
\end{array}\right]\left(\begin{array}{cc}
a & m \\
& b
\end{array}\right) & =\left[\begin{array}{cc}
x \circ a-a \circ x & x \circ m-m \circ z \\
z \circ b-b \circ z
\end{array}\right] \\
& =\left[\begin{array}{cc}
0 & \phi(m) \\
0
\end{array}\right] \\
& =\Delta_{\phi}\left(\begin{array}{cc}
a & m \\
& b
\end{array}\right) .
\end{aligned}
$$

Hence $\Delta_{\phi}$ is inner.

Conversely, suppose $\Delta_{\phi}$ is inner, say $\Delta_{\phi}=\delta\left[\begin{array}{ll}x & y \\ & z\end{array}\right]$ with $\left[\begin{array}{ll}x & y \\ & z\end{array}\right] \in$ $\mathcal{T}^{(2 n)}$. Then

$$
\begin{aligned}
\delta\left[\begin{array}{ll}
x & y \\
& z
\end{array}\right]\left(\begin{array}{cc}
z & m \\
& b
\end{array}\right) & =\left[\begin{array}{cc}
x \circ a-a \circ x & x \circ m-m \circ z+y \circ b-a \circ y \\
z \circ b-b \circ z
\end{array}\right] \\
& =\left[\begin{array}{cc}
0 & \phi(m) \\
0
\end{array}\right] .
\end{aligned}
$$

Hence $x \in Z_{\mathcal{A}}\left(\mathcal{A}, \mathcal{A}^{(2 n)}\right)$ and $z \in Z_{\mathcal{B}}\left(\mathcal{B}, \mathcal{B}^{(2 n)}\right)$. Moreover, $\phi(m)=x \circ m-$ $m \circ z+y \circ b-a \circ y$. Since $\phi \in \operatorname{Hom}_{\mathcal{A}, \mathcal{B}}\left(\mathcal{M}, \mathcal{M}^{(2 n)}\right)$, it follows that $y \circ b-$ $a \circ y=0$ and so $\phi(m)=x \circ m-m \circ z=\rho_{x, z}(m)$ for all $m \in \mathcal{M}$. Thus $\phi \in Z R_{\mathcal{A}, \mathcal{B}}\left(\mathcal{M}, \mathcal{M}^{(2 n)}\right)$, as claimed.

The next theorem is a direct analogue of [7], Thm. 2.8.

3.12. Theorem. Let $\mathcal{A}$ and $\mathcal{B}$ be unital Banach algebras and $\mathcal{M}$ be a unital Banach $\mathcal{A}, \mathcal{B}$-module. Let $\mathcal{T}=\left[\begin{array}{cc}\mathcal{A} & \mathcal{M} \\ & \mathcal{B}\end{array}\right]$ be the corresponding triangular Banach algebra. If $n$ is a positive integer and both $\mathcal{A}$ and $\mathcal{B}$ are $(2 n)$-weakly amenable, then

$$
H^{1}\left(\mathcal{T}, \mathcal{T}^{(2 n)}\right) \simeq \operatorname{Hom}_{\mathcal{A}, \mathcal{B}}\left(\mathcal{M}, \mathcal{M}^{(2 n)}\right) / Z R_{\mathcal{A}, \mathcal{B}}\left(\mathcal{M}, \mathcal{M}^{(2 n)}\right) .
$$

Proof. Let $\Theta: \operatorname{Hom}_{\mathcal{A}, \mathcal{B}}\left(\mathcal{M}, \mathcal{M}^{(2 n)}\right) \rightarrow H^{1}\left(\mathcal{T}, \mathcal{T}^{(2 n)}\right)$ be defined by $\Theta(\phi)=\overline{\Delta_{\phi}}$, where $\overline{\Delta_{\phi}}$ denotes the equivalence class of $\Delta_{\phi}$ in $H^{1}\left(\mathcal{T}, \mathcal{T}^{(2 n)}\right)$. Then it is relatively simple to verify that $\Theta$ is linear.

To see that $\Theta$ is surjective, let $\delta: \mathcal{T} \rightarrow \mathcal{T}^{(2 n)}$ be a continuous derivation. By Proposition 3.9, we can find derivations $\delta_{1}: \mathcal{A} \rightarrow \mathcal{A}^{(2 n)}, \delta_{4}: \mathcal{B} \rightarrow \mathcal{B}^{(2 n)}$, a linear map $\rho: \mathcal{M} \rightarrow \mathcal{M}^{(2 n)}$ and an element $\gamma_{\delta} \in \mathcal{M}^{(2 n)}$ such that

$$
\delta\left(\begin{array}{cc}
a & m \\
& b
\end{array}\right)=\left[\begin{array}{cc}
\delta_{1}(a) & a \circ \gamma_{\delta}-\gamma_{\delta} \circ b+\rho(m) \\
\delta_{4}(b)
\end{array}\right] .
$$

Our assumption that $\mathcal{A}$ and $\mathcal{B}$ are $(2 n)$-weakly amenable implies the existence of $x \in \mathcal{A}^{(2 n)}, z \in \mathcal{B}^{(2 n)}$ such that $\delta_{1}=\delta_{x}$ and $\delta_{4}=\delta_{z}$. Define $\delta_{0}: \mathcal{T} \rightarrow \mathcal{T}^{(2 n)}$ via

$$
\delta_{0}\left(\begin{array}{cc}
a & m \\
& b
\end{array}\right)=\left[\begin{array}{cc}
\delta_{x}(a) & \rho_{x, z}(m)+a \circ \gamma_{\delta}-\gamma_{\delta} \circ b \\
\delta_{z}(b)
\end{array}\right]
$$


Then $\delta_{0}=\delta\left[\begin{array}{cc}x & -\gamma_{\delta} \\ z\end{array}\right]$ is inner, and hence $\delta_{1}=\delta-\delta_{0}$ satisfies $\overline{\delta_{1}}=\bar{\delta}$ in $H^{1}\left(\mathcal{T}, \mathcal{T}^{(2 n)}\right)$. Furthermore, $\delta_{1}\left(\begin{array}{cc}a & m \\ & b\end{array}\right)=\left[\begin{array}{cc}0 & \rho_{1}(m) \\ 0\end{array}\right]$, where $\rho_{1}=\rho-\rho_{x, z}$. Since $\rho, \rho_{x, z}$ belong to $\operatorname{Hom}_{\mathcal{A}, \mathcal{B}}\left(\mathcal{M}, \mathcal{M}^{(2 n)}\right)$, so does $\rho_{1}$. By Proposition 3.9 (ii), $\Theta\left(\rho_{1}\right)=\overline{\Delta_{\rho_{1}}}=\overline{\delta_{1}}=\bar{\delta}$, showing that $\Theta$ is indeed onto.

Next, $\phi \in \operatorname{ker} \Theta$ precisely when $\Theta(\phi)$ is inner. Again, by Lemma 3.11 this is equivalent to the assertion that $\phi \in Z R_{\mathcal{A}, \mathcal{B}}\left(\mathcal{M}, \mathcal{M}^{(2 n)}\right)$. It then follows that

$$
\begin{aligned}
\operatorname{ran} \Theta=H^{1}\left(\mathcal{T}, \mathcal{T}^{(2 n)}\right) & \simeq \operatorname{Hom}_{\mathcal{A}, \mathcal{B}}\left(\mathcal{M}, \mathcal{M}^{(2 n)}\right) / \operatorname{ker} \Theta \\
& =\operatorname{Hom}_{\mathcal{A}, \mathcal{B}}\left(\mathcal{M}, \mathcal{M}^{(2 n)}\right) / Z R_{\mathcal{A}, \mathcal{B}}\left(\mathcal{M}, \mathcal{M}^{(2 n)}\right)
\end{aligned}
$$

\section{EXAMPLES}

4.1. Example One: $\mathcal{T}_{2} \otimes \mathcal{A}$, where $\mathcal{A}$ is a unital $C^{*}$-algebra. Suppose $\mathcal{B}$ is a unital Banach algebra and consider $\mathcal{T}_{\mathcal{B}}=\mathcal{T}_{2} \otimes \mathcal{B}$. By Theorem 3.4, $H^{1}\left(\mathcal{T}_{\mathcal{B}}, \mathcal{T}_{\mathcal{B}}^{(2 n-1)}\right)$ $\simeq H^{1}\left(\mathcal{B}, \mathcal{B}^{(2 n-1)}\right) \oplus H^{1}\left(\mathcal{B}, \mathcal{B}^{(2 n-1)}\right)$. In the case where $\mathcal{A}$ is a unital $C^{*}$-algebra, Theorem 3.1 of [5] states that $\mathcal{A}$ is permanently weakly amenable, and hence if we set $\mathcal{T}=\mathcal{T}_{2} \otimes \mathcal{A}$, then $H^{1}\left(\mathcal{T}, \mathcal{T}^{(2 n-1)}\right) \simeq 0 \oplus 0=0$. That is, $\mathcal{T}$ is $(2 n-1)$-weakly amenable for all positive integers $n$.

As for $(2 n)$-weak amenability of $\mathcal{T}$, we first recall the following result.

4.2. Proposition (5], Cor. 1.12). Let $\mathcal{B}$ be a Banach algebra such that $\mathcal{B}^{(2 n)}$ is Arens regular, and suppose that $H^{1}\left(\mathcal{B}^{(2 n+2)}, \mathcal{B}^{(2 n+2)}\right)=0$ for each positive integer $n$. Then $\mathcal{B}$ is $(2 n)$-weakly amenable for each $n \in \mathbb{N}$.

It is well-known that every unital $C^{*}$-algebra $\mathcal{A}$ is Arens regular [4, and thus so is $\mathcal{A}^{(2 n)}, n \geq 1$, since each $\mathcal{A}^{(2 n)}$ is a unital von Neumann algebra. By Proposition 2.3 each $\mathcal{T}^{(2 n)}=\mathcal{T}_{2} \otimes \mathcal{A}^{(2 n)}$ is Arens regular, and in order to apply Proposition 4.2 to $\mathcal{T}$, it suffices to show that

$$
H^{1}\left(\mathcal{T}^{(2 n+2)}, \mathcal{T}^{(2 n+2)}\right)=0
$$

for each $n \in \mathbb{Z}^{+}$. We first require a result which is in fact the converse of 7], Proposition 3.3. That result in turn depends on simple algebraic lemma, the following whose proof is left to the reader.

4.3. Lemma. Let $V$ be a unital algebra. Then $H_{o m}(V) \simeq Z(V) \simeq Z R_{V}(V)$, where $\operatorname{Hom}_{V}(V)$ denotes the $V$-module maps from $V$ into itself, $Z(V)$ denotes the centre of $V$, and $Z R_{V}=\left\{\rho_{x, z}: V \rightarrow V ; x, z \in Z(V)\right\}$.

4.4. Proposition. Let $\mathcal{B}$ be a unital Banach algebra. Then $H^{1}(\mathcal{B}, \mathcal{B})=0$ if and only if $H^{1}\left(\mathcal{T}_{2} \otimes \mathcal{B}, \mathcal{T}_{2} \otimes \mathcal{B}\right)=0$.

Proof. If $H^{1}\left(\mathcal{T}_{2} \otimes \mathcal{B}, \mathcal{T}_{2} \otimes \mathcal{B}\right)=0$, then $H^{1}(\mathcal{B}, \mathcal{B})=0$ by Proposition 3.3 of [7]. If $H^{1}(\mathcal{B}, \mathcal{B})=0$, then by Theorem 2.8 of the same paper,

$$
\begin{aligned}
H^{1}\left(\mathcal{T}_{2} \otimes \mathcal{B}, \mathcal{T}_{2} \otimes \mathcal{B}\right) & \simeq \operatorname{Hom}_{\mathcal{B}}(\mathcal{B}) / Z R_{\mathcal{B}}(\mathcal{B}) \\
& =0
\end{aligned}
$$

by Lemma 4.3 above. 
Equipped with the above proposition, we can now complete our analysis of $H^{1}\left(\mathcal{T}^{(2 n)}, \mathcal{T}^{(2 n)}\right)$. Indeed, since $\mathcal{A}^{(2 n+2)}$ is a unital von Neumann algebra for each positive integer $n$, a theorem of Sakai [14] (see also Kadison 11] for an independent proof) asserts that each $H^{1}\left(\mathcal{A}^{(2 n+2)}, \mathcal{A}^{(2 n+2)}\right)=0$. Proposition 4.4 then shows that $H^{1}\left(\mathcal{T}^{(2 n+2)}, \mathcal{T}^{(2 n+2)}\right)=0$, which is, as pointed out earlier, sufficient to prove that $\mathcal{T}$ is $(2 n)$-weakly amenable for all natural numbers $n$.

Summarizing:

4.5. Proposition. If $\mathcal{A}$ is a unital $C^{*}$-algebra, then $\mathcal{T}=\mathcal{T}_{2} \otimes \mathcal{A}$ is permanently weakly amenable.

4.6. Example Two: odd versus even weak amenability. Our next example shows that permanent weak amenability is not guaranteed by the fact that the corner algebras are themselves permanently weakly amenable.

Let $\mathcal{H}$ be an infinite dimensional, complex, separable Hilbert space, let $\mathcal{B}(\mathcal{H})$ denote the set of bounded linear operators on $\mathcal{H}$, and let $\mathcal{K}(\mathcal{H})$ denote the closed, two-sided ideal of compact operators in $\mathcal{B}(\mathcal{H})$. We set $\mathcal{Q}(\mathcal{H})$ to denote the quotient algebra $\mathcal{B}(\mathcal{H}) / \mathcal{K}(\mathcal{H})$, and let $\pi: \mathcal{B}(\mathcal{H}) \rightarrow \mathcal{Q}(\mathcal{H})$ denote the canonical quotient map. Consider the algebra $\mathcal{A}=\mathbb{C} I+\mathcal{K}(\mathcal{H})$, the unitization of $\mathcal{K}(\mathcal{H})$, and let

$$
\mathcal{T}=\left[\begin{array}{cc}
\mathcal{A} & \mathcal{B}(\mathcal{H}) \\
& \mathcal{A}
\end{array}\right]
$$

As we saw in the previous example, since $\mathcal{A}$ is a unital $C^{*}$-algebra, [5], Theorem 3.1 implies that $\mathcal{A}$ is permanently weakly amenable. Applying Theorem [3.7, we obtain

$$
\mathcal{T} \text { is }(2 n-1) \text {-weakly amenable for all } n \geq 1 \text {. }
$$

As we shall now see, $\mathcal{T}$ is not 2 -weakly amenable (and hence $\mathcal{T}$ is not $(2 n)$-weakly amenable for any $n \geq 1$, by [5], Proposition 1.2). The proof is an application of Theorem 3.12. Namely,

$$
H^{1}\left(\mathcal{T}, \mathcal{T}^{(2)}\right) \simeq \operatorname{Hom}_{\mathcal{A}, \mathcal{A}}\left(\mathcal{B}(\mathcal{H}), \mathcal{B}(\mathcal{H})^{(2)}\right) / Z R_{\mathcal{A}, \mathcal{A}}\left(\mathcal{B}(\mathcal{H}), \mathcal{B}(\mathcal{H})^{(2)}\right)
$$

Before we begin, let us briefly describe $\mathcal{B}(\mathcal{H})^{(2)}$, as well as the action of $\mathcal{B}(\mathcal{H})$ and $\mathcal{A}$ upon $\mathcal{B}(\mathcal{H})^{(2)}$. Recall that a closed subspace $\mathfrak{Y}$ of a Banach space $\mathfrak{X}$ is called an $M$-ideal if there is a linear projection $\eta: \mathfrak{X}^{*} \rightarrow \mathfrak{Y}^{\perp}$, where $\mathfrak{Y}^{\perp}=\left\{\psi \in \mathfrak{X}^{*}\right.$ : $\left.\left.\psi\right|_{\mathfrak{Y}}=0\right\}$, and if for each $\phi \in \mathfrak{X}^{*}$ we have $\|\phi\|=\|\eta(\phi)\|+\|\phi-\eta(\phi)\|$. We refer the reader to [6], Chapter 11, for more information regarding $M$-ideals. For our purposes, we shall only require the following facts:

(a) If $\mathfrak{Y}$ is an $M$-ideal of a Banach space $\mathfrak{X}$, then every $y^{*} \in \mathfrak{Y}^{*}$ has a unique Hahn-Banach extension to $\mathfrak{X}^{*}$. This allows us to view $\mathfrak{Y}^{*}$ as a subspace of $\mathfrak{X}^{*}$, and with respect to this embedding, $\mathfrak{X}^{*}=\mathfrak{Y}^{*} \oplus_{1} \mathfrak{Y}^{\perp}$ (i.e. the $\ell^{1}$-direct sum).

(b) A subspace $\mathcal{K}$ of a $C^{*}$-algebra $\mathcal{B}$ is an $M$-ideal if and only if $\mathcal{K}$ is a closed, two-sided ideal of $\mathcal{B}$.

(c) If $\mathcal{B}$ is a $C^{*}$-algebra and $\mathcal{K}$ is a closed, two-sided ideal of $\mathcal{B}$, then $\mathcal{B}^{*} \simeq$ $\mathcal{K}^{*} \oplus_{1} \mathcal{K}^{\perp}$. Hence $\mathcal{B}^{(2)} \simeq \mathcal{K}^{(2)} \oplus_{\infty}\left(\mathcal{K}^{\perp}\right)^{*}$, and both $\mathcal{K}^{(2)}$ and $\left(\mathcal{K}^{\perp}\right)^{*}$ are ideals in $\mathcal{B}^{(2)}$.

Before applying this to our example, recall also that 
(i) $\mathcal{K}(\mathcal{H})^{*}$ is isometrically isomorphic to $\mathcal{C}_{1}(\mathcal{H})$, the trace class operators on $\mathcal{H}$, via the map

$$
\begin{aligned}
\nu: \mathcal{C}_{1}(\mathcal{H}) & \rightarrow \mathcal{K}(\mathcal{H})^{*}, \\
C & \mapsto \phi_{C},
\end{aligned}
$$

where $\phi_{C}(K)=\operatorname{tr}(C K), K \in \mathcal{K}(\mathcal{H})$. Here, $\operatorname{tr}(\cdot)$ represents the trace functional on $\mathcal{C}_{1}(\mathcal{H})$.

(ii) $\mathcal{K}(\mathcal{H})^{(2)} \simeq \mathcal{C}_{1}(\mathcal{H})^{*}$ is isometrically isomorphic to $\mathcal{B}(\mathcal{H})$ via a similar map:

$$
\begin{aligned}
\theta: \mathcal{B}(\mathcal{H}) & \rightarrow \mathcal{C}_{1}(\mathcal{H})^{*}, \\
X & \mapsto \phi_{X},
\end{aligned}
$$

where $\phi_{X}(C)=\operatorname{tr}(X C)$ for each $C \in \mathcal{C}_{1}(\mathcal{H})$.

By (a) and (b) above, we have

$$
\begin{aligned}
\mathcal{B}(\mathcal{H})^{*} & \simeq \mathcal{K}(\mathcal{H})^{*} \oplus_{1} \mathcal{K}(\mathcal{H})^{\perp} \\
& \simeq \mathcal{C}_{1}(\mathcal{H}) \oplus_{1}(\mathcal{B}(\mathcal{H}) / \mathcal{K}(\mathcal{H}))^{*} \\
& \simeq \mathcal{C}_{1}(\mathcal{H}) \oplus_{1} \mathcal{Q}(\mathcal{H})^{*}
\end{aligned}
$$

and

$$
\begin{aligned}
\mathcal{B}(\mathcal{H})^{(2)} & \simeq \mathcal{C}_{1}(\mathcal{H})^{*} \oplus_{\infty} \mathcal{Q}(\mathcal{H})^{(2)} \\
& \simeq \mathcal{B}(\mathcal{H}) \oplus_{\infty} \mathcal{Q}(\mathcal{H})^{(2)}
\end{aligned}
$$

Moreover, $\mathcal{B}(\mathcal{H})$ and $\mathcal{Q}(\mathcal{H})^{(2)}$ are ideals in $\mathcal{B}(\mathcal{H})^{(2)}$.

Consider the action of $\mathcal{K}(\mathcal{H})$ on $\mathcal{B}(\mathcal{H})^{*}$. Given $K \in \mathcal{K}(\mathcal{H})$ and $\phi_{C} \in \mathcal{C}_{1}(\mathcal{H})$, we see that $\left(K \circ \phi_{C}\right)(L)=\phi_{C}(L K)=\operatorname{tr}(C L K)=\operatorname{tr}(K C L)=\phi_{K C}(L)$ for all $L \in \mathcal{K}(\mathcal{H})$, and hence $\left(K \circ \phi_{C}\right)=\phi_{K C}$. Similarly, $\left(\phi_{C} \circ K\right)=\phi_{C K}$. As for the action of $K$ on $\sigma \in \mathcal{Q}(\mathcal{H})^{*},(K \circ \sigma)(a)=\sigma(K \circ a)=\sigma(\pi(K) \cdot a)=\sigma(0 \cdot a)=\sigma(0)=0$ for all $a \in \mathcal{Q}(\mathcal{H})$, and so $K \circ \sigma=0$. Likewise, $\sigma \circ K=0$.

Considering next the action of $\mathcal{K}(\mathcal{H})$ on $\mathcal{B}(\mathcal{H})^{(2)}$, we find that if $\gamma_{X} \in \mathcal{C}_{1}(\mathcal{H})^{*} \simeq$ $\mathcal{B}(\mathcal{H})$, then calculations similar to those above show that $K \circ \gamma_{X}=\gamma_{K X}, \gamma_{X} \circ$ $K=\gamma_{X K}$ for all $K \in \mathcal{K}(\mathcal{H})$. As such, this action is equivalent to usual operator multiplication. From above, if $\beta \in \mathcal{Q}(\mathcal{H})^{(2)}$, then $(K \circ \beta)(\sigma)=\beta(\sigma \circ K)=\beta(0)=$ $0=\beta(K \circ \sigma)=(\beta \circ K)(\sigma)$. Thus $\beta \circ K=K \circ \beta=0$ for all $K \in \mathcal{K}(\mathcal{H})$ and $\beta \in \mathcal{Q}(\mathcal{H})^{(2)}$, so that $\mathcal{K}(\mathcal{H})$ acts trivially on $\mathcal{Q}(\mathcal{H})^{(2)}$.

With this background, we can (finally!) compute $\operatorname{Hom}_{\mathcal{A}, \mathcal{A}}\left(\mathcal{B}(\mathcal{H}), \mathcal{B}(\mathcal{H})^{(2)}\right)$.

Given $\Phi: \mathcal{B}(\mathcal{H}) \rightarrow \mathcal{B}(\mathcal{H})^{(2)} \simeq \mathcal{B}(\mathcal{H}) \oplus \mathcal{Q}(\mathcal{H})^{(2)}$, we may decompose $\Phi$ as $\left(\Phi_{1}, \Phi_{2}\right)$, where

$$
\Phi_{1}(X)=P_{1}(X) \quad \text { and } \quad \begin{aligned}
P_{1}: \quad \mathcal{B}(\mathcal{H})^{(2)} & \rightarrow \mathcal{B}(\mathcal{H}) \\
(Z, \beta) & \mapsto \quad Z
\end{aligned}
$$

while

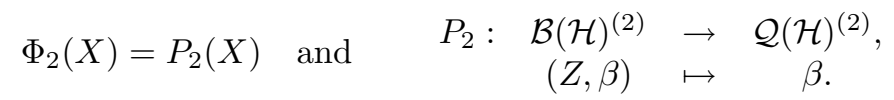

Since both $\mathcal{B}(\mathcal{H})$ and $\mathcal{Q}(\mathcal{H})^{(2)}$ are ideals in $\mathcal{B}(\mathcal{H})^{(2)}$, it follows that

$$
\Phi \in \operatorname{Hom}_{\mathcal{A}, \mathcal{A}}\left(\mathcal{B}(\mathcal{H}), \mathcal{B}(\mathcal{H})^{(2)}\right)
$$

if and only if

$$
\Phi_{1} \in H o m_{\mathcal{A}, \mathcal{A}}(\mathcal{B}(\mathcal{H}), \mathcal{B}(\mathcal{H})) \quad \text { and } \quad \Phi_{2} \in H o m_{\mathcal{A}, \mathcal{A}}\left(\mathcal{B}(\mathcal{H}), \mathcal{Q}(\mathcal{H})^{(2)}\right) .
$$


That is,

$$
\operatorname{Hom}_{\mathcal{A}, \mathcal{A}}\left(\mathcal{B}(\mathcal{H}), \mathcal{B}(\mathcal{H})^{(2)}\right) \simeq \operatorname{Hom}_{\mathcal{A}, \mathcal{A}}(\mathcal{B}(\mathcal{H}), \mathcal{B}(\mathcal{H})) \oplus \operatorname{Hom}_{\mathcal{A}, \mathcal{A}}\left(\mathcal{B}(\mathcal{H}), \mathcal{Q}(\mathcal{H})^{(2)}\right)
$$

But $\operatorname{Hom}_{\mathcal{A}, \mathcal{A}}(\mathcal{B}(\mathcal{H}), \mathcal{B}(\mathcal{H}))=\operatorname{Hom}_{\mathcal{K}(\mathcal{H}), \mathcal{K}(\mathcal{H})}(\mathcal{B}(\mathcal{H}), \mathcal{B}(\mathcal{H})) \simeq \mathbb{C}$, as is readily verified.

We claim that $\operatorname{Hom}_{\mathcal{A}, \mathcal{A}}\left(\mathcal{B}(\mathcal{H}), \mathcal{Q}(\mathcal{H})^{(2)}\right) \simeq \mathcal{B}\left(\mathcal{Q}(\mathcal{H}), \mathcal{Q}(\mathcal{H})^{(2)}\right)$, the bounded linear operators from the Calkin algebra into its second dual. To see this, suppose that $\phi \in \mathcal{B}\left(\mathcal{Q}(\mathcal{H}), \mathcal{Q}(\mathcal{H})^{(2)}\right)$, and let $\Phi=\phi \circ \pi \in \mathcal{B}\left(\mathcal{B}(\mathcal{H}), \mathcal{Q}(\mathcal{H})^{(2)}\right)$, the space of bounded linear operators from $\mathcal{B}(\mathcal{H})$ into $\mathcal{Q}(\mathcal{H})^{(2)}$. If $\lambda \in \mathbb{C}, K \in \mathcal{K}(\mathcal{H})$, and $X \in \mathcal{B}(\mathcal{H})$, then

$$
\begin{aligned}
\Phi((\lambda I+K) X) & =\phi(\pi((\lambda I+K) X)) \\
& =\lambda \phi(\pi(X)) \\
& =\lambda \Phi(X) \\
& =\Phi(X(\lambda I+K)) .
\end{aligned}
$$

In other words, $\Phi \in \operatorname{Hom}_{\mathcal{A}, \mathcal{A}}\left(\mathcal{B}(\mathcal{H}), \mathcal{Q}(\mathcal{H})^{(2)}\right)$.

It is not hard to verify that the map $\Theta: \phi \mapsto \Phi=\phi \circ \pi$ is linear and injective. It remains to prove that it is surjective. To that end, suppose that $\Psi \in \operatorname{Hom}_{\mathcal{A}, \mathcal{A}}\left(\mathcal{B}(\mathcal{H}), \mathcal{Q}(\mathcal{H})^{(2)}\right)$, and let $K \in \mathcal{K}(\mathcal{H})$. Then $\Psi(K)=\Psi(K \cdot 1)=$ $K \circ \Psi(1)=0$, since the action of $\mathcal{K}(\mathcal{H})$ on $\mathcal{Q}(\mathcal{H})^{(2)}$ is trivial. This means that the map

$$
\begin{aligned}
\psi: \mathcal{Q}(\mathcal{H}) & \rightarrow \mathcal{Q}(\mathcal{H})^{(2)}, \\
\pi(X) & \mapsto \Psi(X),
\end{aligned}
$$

is well-defined and bounded. (Indeed, if $\|\pi(X)\|=1$, we can choose $K \in \mathcal{K}(\mathcal{H})$ such that $\|X+K\|=1$, and then $\|\psi(\pi(X))\|=\|\Psi(X)\|=\|\Psi(X+K)\| \leq\|\Psi\| \| X+$ $K\|=\| \Psi \|$.) Moreover, it is clear that $\Theta(\psi)=\Psi$, and thus $\Theta$ is a linear isomorphism.

So far we have shown that

$$
\operatorname{Hom}_{\mathcal{A}, \mathcal{A}}\left(\mathcal{B}(\mathcal{H}), \mathcal{B}(\mathcal{H})^{(2)}\right) \simeq \mathbb{C} \oplus \mathcal{B}\left(\mathcal{Q}(\mathcal{H}), \mathcal{Q}(\mathcal{H})^{(2)}\right)
$$

Next we must consider $Z R_{\mathcal{A}, \mathcal{A}}(\mathcal{B}(\mathcal{H}), \mathcal{B}(\mathcal{H}))$. Borrowing from our above analysis, $\mathcal{K}(\mathcal{H})$ is an ideal in the $C^{*}$-algebra $\mathcal{A}$, and so

$$
\begin{aligned}
\mathcal{A}^{(2)} & =\mathcal{K}(\mathcal{H})^{(2)} \oplus(\mathcal{A} / \mathcal{K}(\mathcal{H}))^{(2)} \\
& =\mathcal{B}(\mathcal{H}) \oplus \mathbb{C}
\end{aligned}
$$

and both $\mathcal{B}(\mathcal{H})$ and $\mathbb{C}$ are ideals of $\mathcal{A}^{(2)}$. The action of $\mathcal{A}$ on $\mathcal{B}(\mathcal{H})$ is again just usual operator multiplication. As for the action of $\mathcal{A}$ on $\mathbb{C}$, as before it must annihilate $\mathcal{K}(\mathcal{H})$, and so we have

$$
(\lambda I+K) \circ \alpha=\lambda \alpha=\alpha \circ(\lambda I+K)
$$

for all $\lambda, \alpha \in \mathbb{C}$ and $K \in \mathcal{K}(\mathcal{H})$.

This tells us that $(X, \alpha) \in Z_{\mathcal{A}}\left(\mathcal{A}^{(2)}\right)$ implies that $((\lambda I+K) X, \lambda \alpha)=(\lambda I+K) \circ$ $(X, \alpha)=(X, \alpha) \circ(\lambda I+K)=(X(\lambda I+K), \alpha \lambda)$ for all $\lambda \in \mathbb{C}$ and $K \in \mathcal{K}(\mathcal{H})$. Thus $X$ lies in the commutant of $\mathcal{K}(\mathcal{H})$ in $\mathcal{B}(\mathcal{H})$, which is $\mathbb{C} I$, since $\mathcal{K}(\mathcal{H})$ is an irreducible $C^{*}$-algebra [12]. The converse is trivial; namely, if $\kappa, \alpha \in \mathbb{C}$, then $(\kappa I, \alpha) \in Z_{\mathcal{A}}\left(\mathcal{A}^{(2)}\right)$. 
Then $Z R_{\mathcal{A}, \mathcal{A}}\left(\mathcal{B}(\mathcal{H}), \mathcal{B}(\mathcal{H})^{(2)}\right)=\left\{\tau_{A, B}: A, B \in Z_{\mathcal{A}}\left(\mathcal{A}^{(2)}\right)\right\}$, and

$$
\begin{aligned}
\tau_{A, B}(X) & =A \circ X-X \circ B \\
& =\left(\kappa_{A} I, \alpha_{A}\right) \circ X-X \circ\left(\kappa_{B} I, \alpha_{B}\right) \\
& =\left(\kappa_{A} X, \alpha_{A} \pi(X)\right)-\left(\kappa_{B} X, \alpha_{B} \pi(X)\right) \\
& =\left(\left(\kappa_{A}-\kappa_{B}\right) I,\left(\alpha_{A}-\alpha_{B}\right)\right) \circ X .
\end{aligned}
$$

Thus $Z R_{\mathcal{A}, \mathcal{A}}\left(\mathcal{B}(\mathcal{H}), \mathcal{B}(\mathcal{H})^{(2)}\right) \simeq \mathbb{C} \oplus \mathbb{C}$ (with elements of the former set being identified with scalar multiplication operators). Finally,

$$
\begin{aligned}
\operatorname{Hom}_{\mathcal{A}, \mathcal{A}}\left(\mathcal{B}(\mathcal{H}), \mathcal{B}(\mathcal{H})^{(2)}\right) & \simeq \frac{\mathbb{C} \oplus \mathcal{B}\left(\mathcal{Q}(\mathcal{H}), \mathcal{Q}(\mathcal{H})^{(2)}\right)}{\mathbb{C} \oplus \mathbb{C}} \\
& \simeq \mathcal{B}\left(\mathcal{Q}(\mathcal{H}), \mathcal{Q}(\mathcal{H})^{(2)}\right) / \mathbb{C} \neq 0 .
\end{aligned}
$$

Thus, by Theorem 3.12, $\mathcal{T}$ is not 2 -weakly amenable.

4.7. Finite dimensional nest algebras are permanently weakly amenable. Recall that a nest on a Hilbert space $\mathcal{H}$ is a chain $\mathcal{N}$ of closed subspaces of $\mathcal{H}$ which contains $\{0\}$ and $\mathcal{H}$, and which is closed under the operations of taking intersections and closed spans. The corresponding nest algebra $\mathcal{T}(\mathcal{N})=\{T \in$ $\mathcal{B}(\mathcal{H}): T N \subseteq N$ for all $N \in \mathcal{N}\}$.

Our main result here is the following:

4.8. Theorem. Let $\mathcal{T}(\mathcal{N})$ be a nest algebra acting on a finite dimensional, complex Hilbert space $\mathcal{H}$. Then $\mathcal{T}(\mathcal{N})$ is permanently weakly amenable.

Proof. Since $\mathcal{T}(\mathcal{N})$ is finite dimensional, it follows that $\mathcal{T}(\mathcal{N})^{(2 n)}$ is isomorphic to $\mathcal{T}(\mathcal{N})$ as a Banach algebra for each $n \geq 1$. But then

$$
H^{1}\left(\mathcal{T}(\mathcal{N}), \mathcal{T}(\mathcal{N})^{(2 n)}\right) \simeq H^{1}(\mathcal{T}(\mathcal{N}), \mathcal{T}(\mathcal{N}))=0
$$

for all $n \geq 1$ ([3], or [14, when the nest is trivial and $\mathcal{T}(\mathcal{N}) \simeq \mathcal{B}(\mathcal{H})$ ).

As for odd weak amenability, the proof works by induction on the dimension $m$ of the space $\mathcal{H}$ upon which $\mathcal{T}(\mathcal{N})$ acts. Of course, when $m=1, \mathcal{T}(\mathcal{N}) \simeq \mathbb{C}$ is an abelian $C^{*}$-algebra; hence it is amenable (and therefore permanently weakly amenable). Suppose the result holds for all nest algebras acting upon Hilbert spaces of dimension less than $k$. Let $\mathcal{T}(\mathcal{N})$ be a nest algebra acting on a space of dimension $k$. Then, either the nest $\mathcal{N}$ is trivial, in which case $\mathcal{T}(\mathcal{N}) \simeq \mathcal{B}(\mathcal{H})$ is a $C^{*}$-algebra, hence is permanently weakly amenable by [5], Thm. 2.1 , or there exists an element $\{0\} \neq N \neq \mathcal{H}$ in $\mathcal{N}$ and we may decompose $\mathcal{T}(\mathcal{N})$ as

$$
\mathcal{T}(\mathcal{N})=\left[\begin{array}{cc}
\mathcal{A} & \mathcal{M} \\
0 & \mathcal{B}
\end{array}\right] \begin{gathered}
N \\
N^{\perp}
\end{gathered}
$$

where $\mathcal{A}$ is a nest algebra acting upon $N, \mathcal{B}$ is a nest algebra acting upon $N^{\perp}$, and $\mathcal{M}$ is the space of all operators taking $N^{\perp}$ into $N$. By our induction hypothesis, $\mathcal{A}$ and $\mathcal{B}$ are permanently weakly amenable. Applying Theorem 3.7 above, we conclude that $\mathcal{T}(\mathcal{N})$ is $(2 n-1)$-weakly amenable for all $n \geq 1$.

Combining these two results yields our theorem.

Of course, the above theorem begs the question which we have been unable to resolve as of this time, namely: are all nest algebras permanently weakly amenable? 


\section{REFERENCES}

[1] Bade, W.G., Curtis, P.C. and Dales, H.G., Amenability and weak amenability for Beurling and Lipschitz algebras, Proc. London Math. Soc. 55 (1987), 359-377. MR 88f:46098

[2] Bade, W.G., Dales, H.G. and Lykova, Z.A., Algebraic and strong splittings of extensions of Banach algebras, Mem. Amer. Math. Soc., 137 (1999), No. 656. MR 99g:46059

[3] Christensen, E., Derivations of nest algebras, Math. Ann., 229 (1977), 155-161. MR 56:6420

[4] Civin, P. and Yood, B., The second conjugate space of a Banach algebra as an algebra, Pacific J. Math., 11 (1961), 847-870. MR 26:622

[5] Dales, H.G., Ghahramani, F. and Grønbæk, N., Derivations into iterated duals of Banach algebras, Studia Math., 128 (1998), 19-54. MR 99g:46064

[6] Davidson, K.R., Nest Algebras, Pitman Research Notes in Mathematics, no. 191, Longman Scientific and Technical, London, 1988. MR 90f:47062

[7] Forrest, B.E. and Marcoux, L.W., Derivations of triangular Banach algebras, Indiana Univ. Math. J. 45 (1996), 441-462. MR 97m:46079

[8] Gilfeather, F. and Smith, R.R., Cohomology for operator algebras: joins, Amer. J. Math. 116 (1994), 541-561. MR 95d:46077

[9] Haagerup, U., All nuclear $C^{*}$-algebras are amenable, Invent. Math. 74 (1983), 305-319. MR 85g:46074

[10] Johnson, B.E., Cohomology in Banach algebras, Mem. Amer. Math. Soc., 127 (1992). MR 51:11130

[11] Kadison, R.V., Derivations of operator algebras, Ann. Math., 83 (1966), 280-293. MR 33:1747

[12] Murphy, G.J., $C^{*}$-Algebras and Operator Theory, Academic Press, San Diego, 1990. MR 91m:46084

[13] Palmer, T.W., Banach algebras and the general theory of ${ }^{*}$-algebras, Vol. 1, Algebras and Banach Algebras, Cambridge Univ. Press, 1994. MR 95c:46002

[14] Sakai, S., Derivations of $W^{*}$-algebras, Ann. Math., 83 (1966), 273-279. MR 33:1748

Department of Pure Mathematics, University of Waterloo, Waterloo, Ontario, CANADA N2L 3G1

E-mail address: beforres@math.uwaterloo.ca

Department of Mathematical Sciences, University of Alberta, Edmonton, Alberta, Canada T6G 2G1

Current address: Department of Pure Mathematics, University of Waterloo, Waterloo, Ontario, Canada N2L 3G1

E-mail address: L.Marcoux@ualberta.ca 